\title{
IL VOTO NEI QUARTIERI DI ROMA DAL 2000 AL 2018
}

\author{
di FEDERICO TOMASSI
}

Quaderni dell'Osservatorio elettorale n. 80, dicembre 2018, pp. 83-110 
Una precedente versione di questo testo è stata pubblicata sul sito internet del Centro per la Riforma dello Stato e poi presentata al convegno SISP-ITANES-SISE «Elezioni politiche e regionali 2017/18» presso l'Università di Salerno. L'autore è l'unico responsabile di eventuali errori e imprecisioni, ma ringrazia per l'ispirazione e i suggerimenti Fabrizio Barca, Alessandro Coppola, Keti Lelo, Salvatore Monni, Walter Tocci, Salvatore Vassallo, Cristiano Vezzoni e i due anonimi referee, oltre ai partecipanti al Laboratorio Roma del CRS, al progetto «Mappa il PD», al convegno «Roma in transizione» del Gran Sasso Science Institute e al blog \#mapparoma.

Abstract - Social and economic inequalities between the city centre and peripheral belts persist for many years in Rome districts, in parallel to the on-going suburban sprawl. That yields political effects too, since electoral results from 2000 to 2018 highlight sharp political polarization: votes for left-wing (right-wing or Five Star Movement) candidates are inversely (directly) proportional to distance from Capitoline Hill. Notably, in 2018 general election, the left-wing coalition prevailed in central districts only, where education levels and personal income are very high, and (even if only slightly) in historical periphery, where it keeps consensus on progressive, inclusive and liberal policies. The opposite holds for the new populist Five Star Movement, that prevailed in low-density periphery inside the Orbital, in far-off sprawled districts and in Ostia coastal area. However, the right-wing coalition won in Rome as a whole, by more homogeneous and even inter-classist consensus, and prevailed outside the Orbital thanks to the League, that had for the very first time many votes in the Roman peripheries. As a matter of facts, both right-wing and populist policies seem easier to the extent that suburban citizens feel deprived since harmed by economic crisis, unsafe and worried about immigrants, far from elected bodies, scarcely endowed with public services.

Keywords: Rome, elections, peripheries, neighbourhoods, inequalities 


\section{Introduzione: le disuguaglianze a Roma}

Molti cambiamenti sono avvenuti a Roma negli ultimi 15-20 anni, dopo che negli anni 90 è iniziata la crisi della pubblica amministrazione, delle partecipazioni pubbliche e della spesa pubblica, il motore tradizionale di crescita della città (Diletti, 2016). Le giunte di centrosinistra guidate da Rutelli e Veltroni hanno cercato di attivare un processo di cambiamento strutturale basato sull'economia della conoscenza e orientato verso le nuove tecnologie, il turismo di massa, la finanza, i servizi avanzati, l'audiovisivo, la cultura e la ricerca (De Muro et al., 2011, p. 1213). Almeno fino allo scoppio della crisi economica globale, questo modello ha effettivamente portato risultati positivi, in termini di crescita del PIL, reddito pro capite e flussi turistici, compensando il minore ruolo pubblico (Causi, 2018).

Tuttavia - come in altre metropoli occidentali - sono proprio le nuove attività legate al paradigma culturale e cognitivo, insieme ai processi di riqualificazione urbana nelle aree centrali, a rendere più attrattive alcune zone urbane, lasciando crescere nelle periferie stagnanti fattori di disagio e antagonismo (Camagni, 2016). A Roma si registrano infatti forti disuguaglianze e polarizzazioni tra centro e periferie (Alleva, 2017; Barca, 2015; Commissione Parlamentare Periferie, 2017, pp. 261-331; De Muro, 2013; De Muro et al., 2011; 2012; Lelo, 2015; Lelo et al., 2017; Violante, 2008), soprattutto nel settore orientale (Cipollini e Truglia, 2015) e sul litorale di Ostia (Violante, 2013). In primo luogo, i benefici sociali ed economici della crescita e i costi della crisi non si sono ripartiti omogeneamente sul territorio, tanto che l'indice di Gini di concentrazione del reddito mostra il Lazio (fortemente influenzato da quanto accade a Roma) molto vicino alle regioni meno sviluppate del sud Italia, e non a quelle del nord (Acciari e Mocetti, 2013). In secondo luogo, lo sviluppo edilizio è avvenuto a parità di popolazione creando sprawl urbano e diseguale dotazione di beni comuni e servizi pubblici nei vari quartieri. Seguendo la suddivisione in fasce urbane proposta da Tocci (2008, pp. 74-109) e basata sulla densità di popolazione del tessuto edilizio e sull'epoca di costruzione ${ }^{1}$, tra il 2002 e il 2017 il centro, la periferia storica e Ostia hanno perso oltre 1' $8 \%$ dei residenti, la periferia anulare ha guadagnato il

${ }^{1}$ (i) Il centro all'interno dell'anello ferroviario più la zona direzionale dell'Eur; (ii) la periferia storica densa, intensiva e popolare costruita tra gli anni 30 e i 70 fino alla prima circonvallazione (Togliatti-Olimpica-Newton); (iii) la periferia anulare estensiva del ceto medio edificata soprattutto tra la metà degli anni Settanta e la metà dei Novanta, più alcune borgate ufficiali o informali, tra la circonvallazione e il GRA; (iv) i quartieri del litorale di Ostia, con caratteristiche da centro urbano a se stante; (v) la variegata periferia esterna al GRA fino al confine comunale. 
$2 \%$ e la periferia esterna al GRA registra addirittura il $40 \%$ di popolazione in più (cfr. Tab. 1 e Fig. 1). In terzo luogo, in alcune aree si sommano problemi di varia natura e si concentrano criticità irrisolte (case popolari, scarsa accessibilità con il trasporto pubblico, gestione dei rifiuti e del verde, centri per migranti, campi rom, prostituzione, degrado estetico), peraltro aggravate dalla corruzione emersa per alcuni di questi temi con le inchieste giudiziarie sul "Mondo di mezzo". Considerazioni simili possono essere estese ai comuni dell'hinterland romano, in particolare alla prima cintura (Lelo et al., 2018).

Le diseguaglianze socio-economiche e territoriali influiscono inevitabilmente anche su idee e scelte politiche, con una netta polarizzazione elettorale tra il centro e le diverse fasce periferiche (Di Padova, 2016; Tomassi, 2013; 2015; Truglia, 2015). Rispetto agli anni Settanta e Ottanta si è consumato, non solo a Roma, un ribaltamento elettorale tra sinistra e destra: «Il consenso della sinistra è passato dai quartieri più distanti a quelli più vicini al centro, dai luoghi meno densi a quelli più densi, dalle zone di trasformazione a quelle di consolidamento» (Tocci, 2015, p. 26).

TAB. 1 - Popolazione e densità a Roma, per fascia urbana.

\begin{tabular}{lrrrrrr}
\hline Area urbana & $\begin{array}{c}\text { Superficie } \\
\text { (ettari) }\end{array}$ & $\begin{array}{c}\text { Popolazione } \\
\text { dic. } 2002\end{array}$ & $\begin{array}{c}\text { Popolazione } \\
\text { dic. } 2017\end{array}$ & $\begin{array}{c}\text { Densità } \\
\text { dic. } \\
2002 \\
\text { (residentiper } \\
\text { ettaro) }\end{array}$ & $\begin{array}{c}\text { Densità } \\
\text { dic. } \\
2017\end{array}$ & $\begin{array}{c}\text { Variazione } \\
2002-17\end{array}$ \\
\hline $\begin{array}{l}\text { Centro + Eur } \\
\begin{array}{l}\text { Periferia } \\
\text { storica }\end{array}\end{array}$ & 7.388 & 424.695 & 391.229 & 57 & 53 & $-8,5$ \\
$\begin{array}{l}\text { Periferia } \\
\text { anulare }\end{array}$ & 11.366 & 1.268 .840 & 1.174 .814 & 112 & 103 & $-8,3$ \\
$\begin{array}{l}\text { Ostia } \\
\text { Periferia }\end{array}$ & 16.219 & 503.565 & 515.798 & 31 & 32 & 1,9 \\
extra-GRA & 2.234 & 89.266 & 83.069 & 40 & 37 & $-7,8$ \\
\hline Totale Roma & 128,530 & 2.802 .500 & 2.876 .614 & 22 & 22 & 39,5 \\
\hline
\end{tabular}




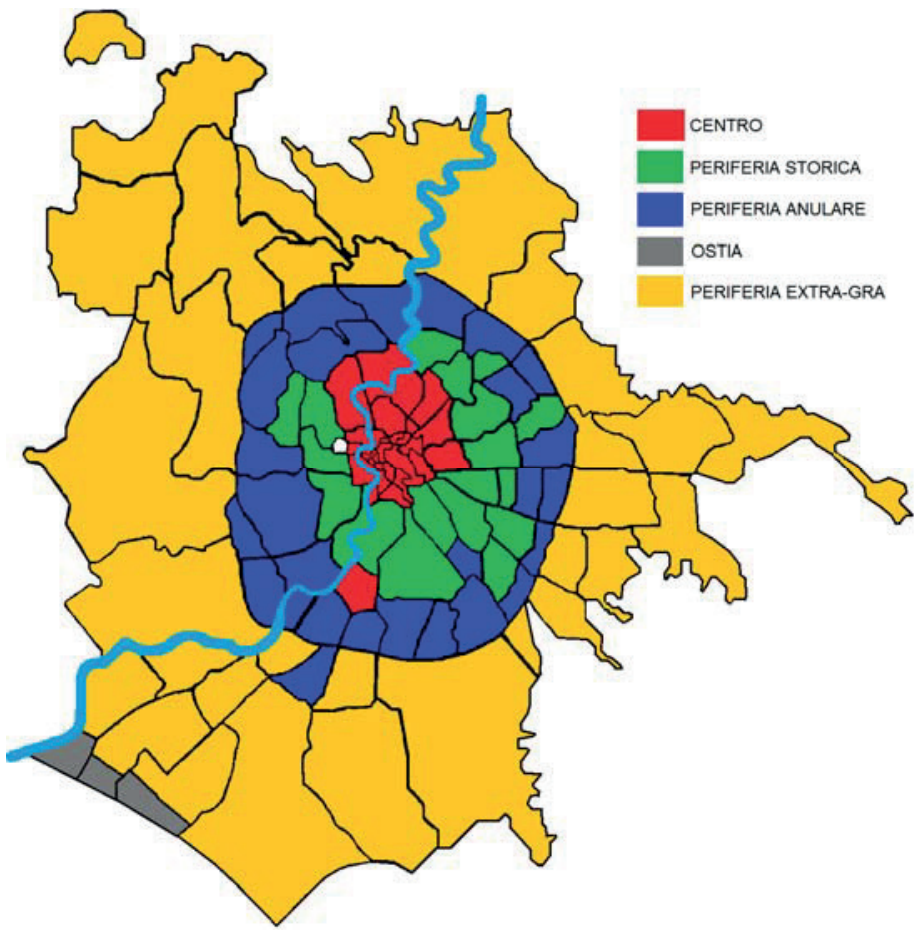

FIG. 1 - Fasce urbane di Roma.

L'obiettivo del presente lavoro è quello di individuare come la distribuzione spaziale delle disuguaglianze socio-economiche influenza i risultati elettorali tra il 2000 e il 2018 nei diversi quartieri romani. L'unità di analisi sono le 132 zone urbanistiche che comprendono seggi elettorali (rispetto alle 155 in cui è suddiviso il territorio comunale, che identificano approssimativamente i quartieri di Roma), per le quali è stato raccolto un dataset con variabili territoriali, demografiche, sociali, economiche e infrastrutturali, che combina dati di diversa fonte: l'anagrafe comunale, i censimenti Istat, i risultati elettorali per sezione e indagini specifiche sui servizi pubblici e privati (Tomassi, 2013; Lelo et al., 2017) ${ }^{2}$.

Il lavoro è organizzato come segue. Nel paragrafo 2 vengono riportati le tendenze di fondo nei dati elettorali dal 2000 al 2018, nel

\footnotetext{
2 I dati utilizzati per le analisi sono disponibili in formato aperto su http://mapparoma.blogspot.it/p/fonti.html; grafici e tabelle sono elaborazioni dell'autore (tranne le mappe che sono di Keti Lelo) su dati di Roma Capitale www.elezioni.comune.roma.it; per il dettaglio delle zone urbanistiche, considerate nell'analisi solo se hanno più di 1000 votanti, si rimanda a http://mapparoma.blogspot.com/p/zone-urbanistiche.html.
} 
complesso della città e nelle diverse fasce urbane. Nei paragrafi 3 e 4 sono approfonditi i risultati del 2018 alle elezioni politiche e regionali, con il dettaglio delle zone urbanistiche. Nel paragrafo 5 sono analizzati i principali fattori sociali, economici, demografici e urbanistici che concorrono a spiegare le dinamiche del voto. Infine, il paragrafo 6 contiene alcuni spunti conclusivi con possibili esiti dell'analisi sul territorio romano.

\section{Variabilità e continuità nei risultati elettorali a Roma}

Le dinamiche elettorali mostrano elementi di variabilità e al contempo di continuità nelle diverse votazioni dal 2000 al 2018. Variano a ogni elezione l'affluenza, i voti assoluti, le coalizioni e i partiti vincenti, secondo l'appropriatezza dei candidati e il contesto politico nazionale e locale; rimangono invece abbastanza costanti, in ogni elezione dal 2000 in poi, le differenze relative nel consenso elettorale tra le diverse fasce urbane e tra zone urbanistiche, indipendentemente dalla coalizione vincente o dal numero assoluto di voti.

Alle elezioni del 2018 ha votato un milione e mezzo di romani, per un'affluenza del $72 \%$, un dato inferiore rispetto a tutte le elezioni politiche precedenti del 2013 (-6 punti percentuali), 2008 (-8), $2006(-12), 2001(-7)$ e 1996 (-14). È però superiore rispetto alle amministrative degli ultimi 10 anni, poco più delle regionali precedenti nel 2013 ( +2 punti), ma molto più del primo turno e del ballottaggio delle due elezioni comunali precedenti nel 2013 e 2016 (+27 punti sul ballottaggio 2013) (cfr. Fig. 2).

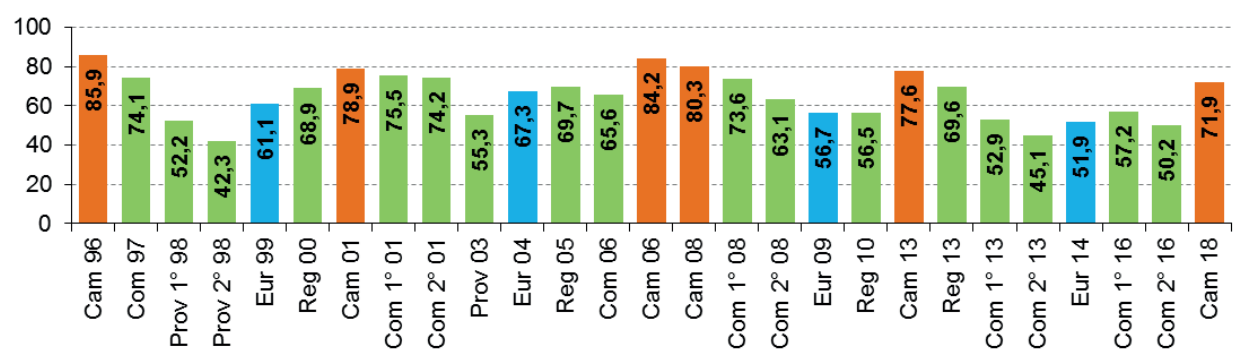

FIG. 2 - Affluenza 1996-2018 (\% degli aventi diritto).

Il calo dell'affluenza che si è manifestato nelle ultime elezioni ha colpito sia il centrosinistra che il centrodestra, anche considerando solo i voti per i candidati presidenti e sindaci (cfr. Fig. 3).

Alle comunali del 2016 (Morcellini et al., 2016), il candidato del PD Giachetti ottiene al primo turno solo 320 mila voti e al secondo 377 mila, che 
rappresentano il punto più basso del centrosinistra dal 2000 in poi. Per il centrodestra, la somma tra i 266mila voti della Meloni e i 141mila di Marchini non è il record negativo (che rimane Alemanno nel 2013 con 364mila voti al primo turno e 375 mila al secondo), ma è comunque pari alla metà rispetto a Tajani 2001 e Alemanno 2008. Il M5S registra un incremento dai 317mila voti di Barillari alle regionali del 2013 ai 454mila della Raggi al primo turno, passando per i soli 150mila di De Vito alle comunali del 2013; è però al ballottaggio che la Raggi fa il pieno di consensi, salendo a 771mila voti nonostante l'affluenza al 50\%: è un risultato molto rilevante, paragonabile a quello di Veltroni nel $2001 \mathrm{e}$ di Alemanno nel 2008, e nettamente superiore a Marino nel 2013. Analizzando i flussi elettorali, si comprende come la neosindaca abbia attratto in parte voti che nelle precedenti elezioni erano andati al centrosinistra, oltre a confermare quasi integralmente i consensi del M5S (Natalizia e Vignati, 2016).

Alle elezioni regionali 2018, la conseguenza della ripresa dell'affluenza è una crescita nei voti assoluti dei candidati di centrosinistra e centrodestra rispetto al recente passato, ma comunque inferiore rispetto ai record degli anni 2000. Zingaretti per il centrosinistra ottiene 545mila voti, mentre cinque anni fa aveva 170 mila voti in più, Parisi per il centrodestra 405mila, poco più di Storace nel 2013, e Lombardi per il M5S 385mila, più di Barillari alle elezioni precedenti ma meno della Raggi, la metà di quanto prese la sindaca al ballottaggio.

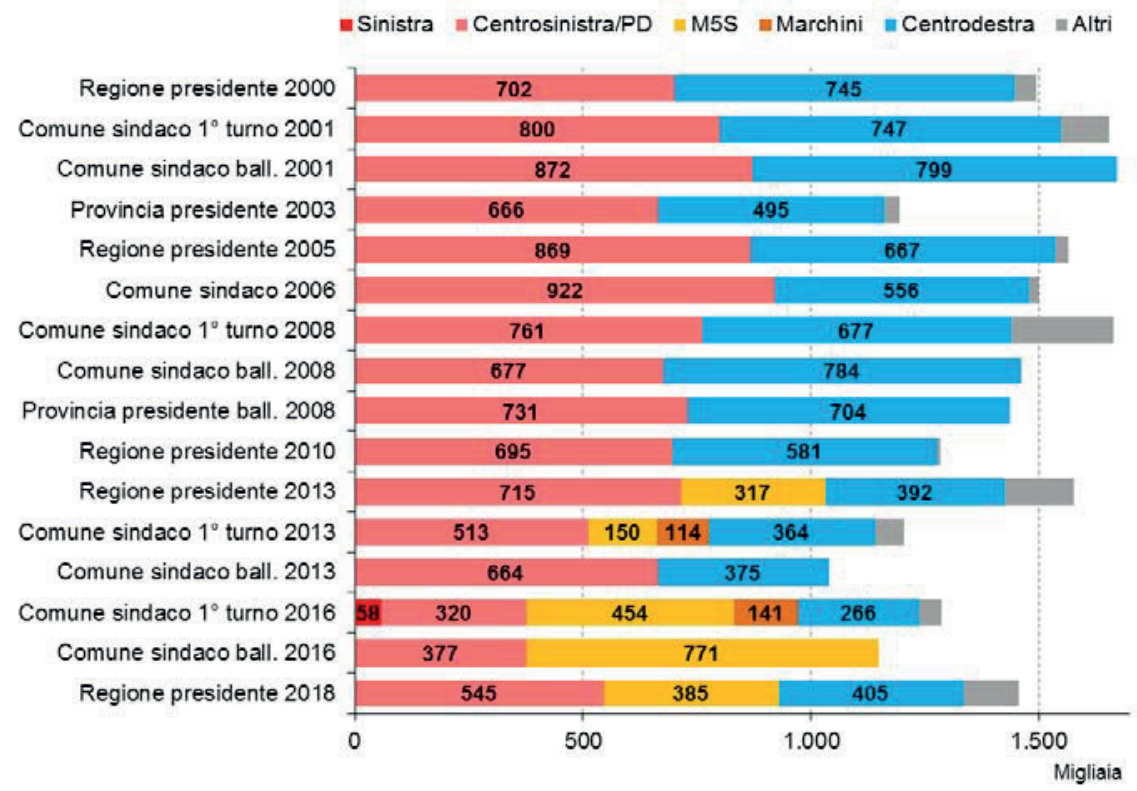

FIG. 3 - Voti assoluti per i candidati delle coalizioni 2000-2018 (migliaia). 
Ma come si distribuiscono questi voti sul territorio romano? In valore assoluto, nel 2018 si tratta di 190mila voti validi nelle zone centrali, 590mila nella periferia storica, 310mila nella periferia anulare, 40mila a Ostia e 328mila nella periferia esterna al GRA (cfr. Tab. 2). La fascia urbana decisiva è quindi ancora la periferia storica (sebbene i suoi residenti siano in continuo calo), dove alle regionali Zingaretti ha ottenuto 100mila voti più di Parisi e Lombardi, mentre alla Camera il centrosinistra ha prevalso di soli 12 mila voti sul centrodestra. Cresce tuttavia l'importanza della periferia esterna al GRA, dove Lombardi ha superato Parisi di $13 \mathrm{mila}$ voti e Zingaretti di 28 mila, ma dove soprattutto alla Camera il M5S ha vinto con 17 mila voti di scarto rispetto al centrodestra e con più del doppio dei voti del centrosinistra.

Vige una sorta di legge gravitazionale, in cui l'attrazione del Campidoglio ha un effetto opposto sui principali schieramenti man mano che ci si allontana verso le periferie (cfr. Tab. 3 e Fig. 4). Le percentuali per il centrosinistra sono inversamente proporzionali alla distanza dal Campidoglio, con il massimo nel centro e nella periferia storica, un valore intermedio nella periferia anulare, e il minimo fuori dal GRA. Viceversa, i voti per il M5S - e in parte per il centrodestra - sono direttamente proporzionali alla distanza dal Campidoglio, con il massimo fuori dal GRA, un valore intermedio nella periferia anulare, e il minimo nella periferia storica e al centro. Ciò vale anche per il voto di lista sia per il PD che per FI o Lega e persino per SEL e LeU, nonché per il referendum costituzionale del 2016, che ha avuto una valenza politica così forte da individuare nel "sì" i caratteri tipici del voto al PD. Nelle analisi che seguono prendiamo a riferimento il centrosinistra perché è l'unica coalizione presente in tutte le elezioni e ballottaggi tenuti nel periodo in esame. 


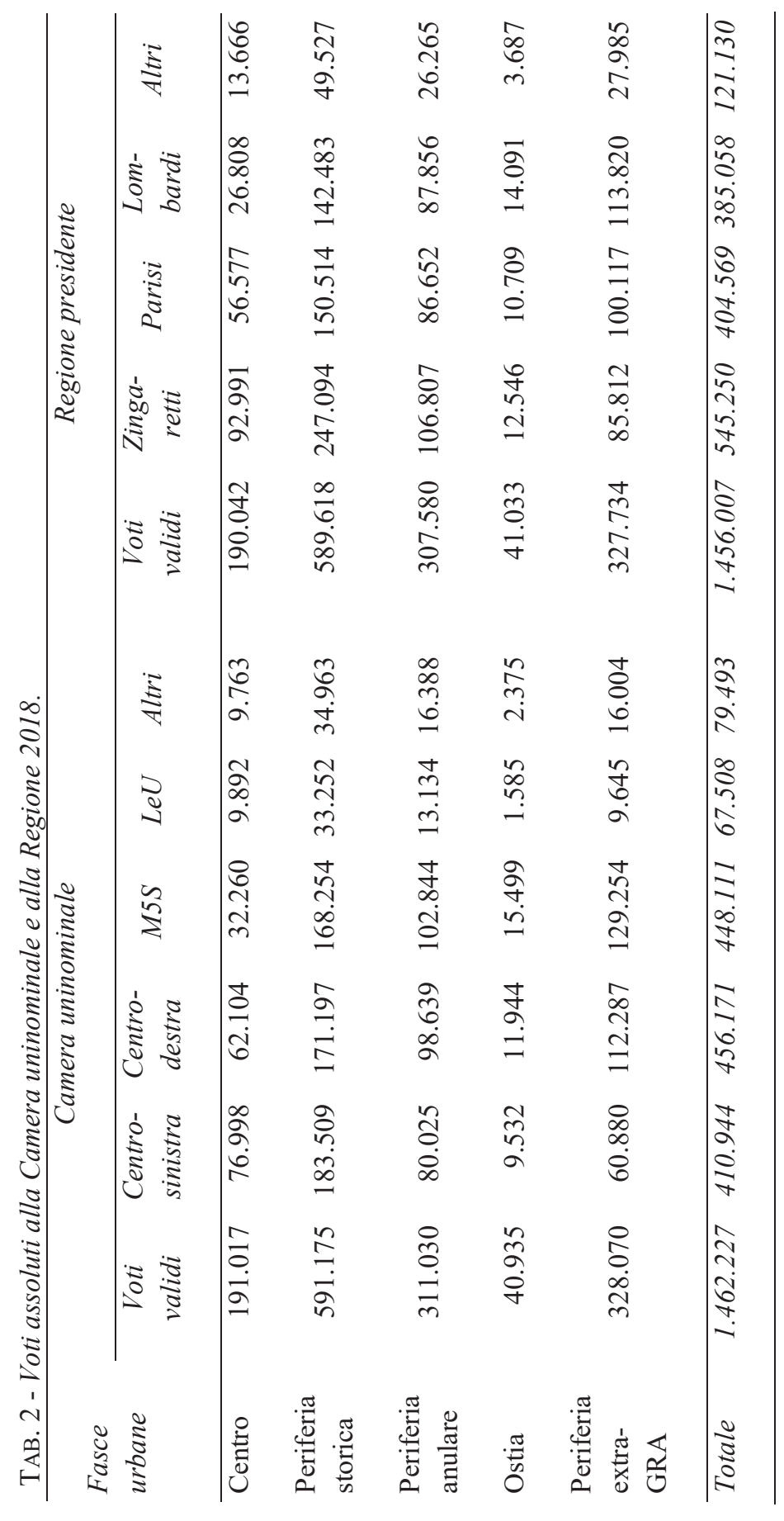




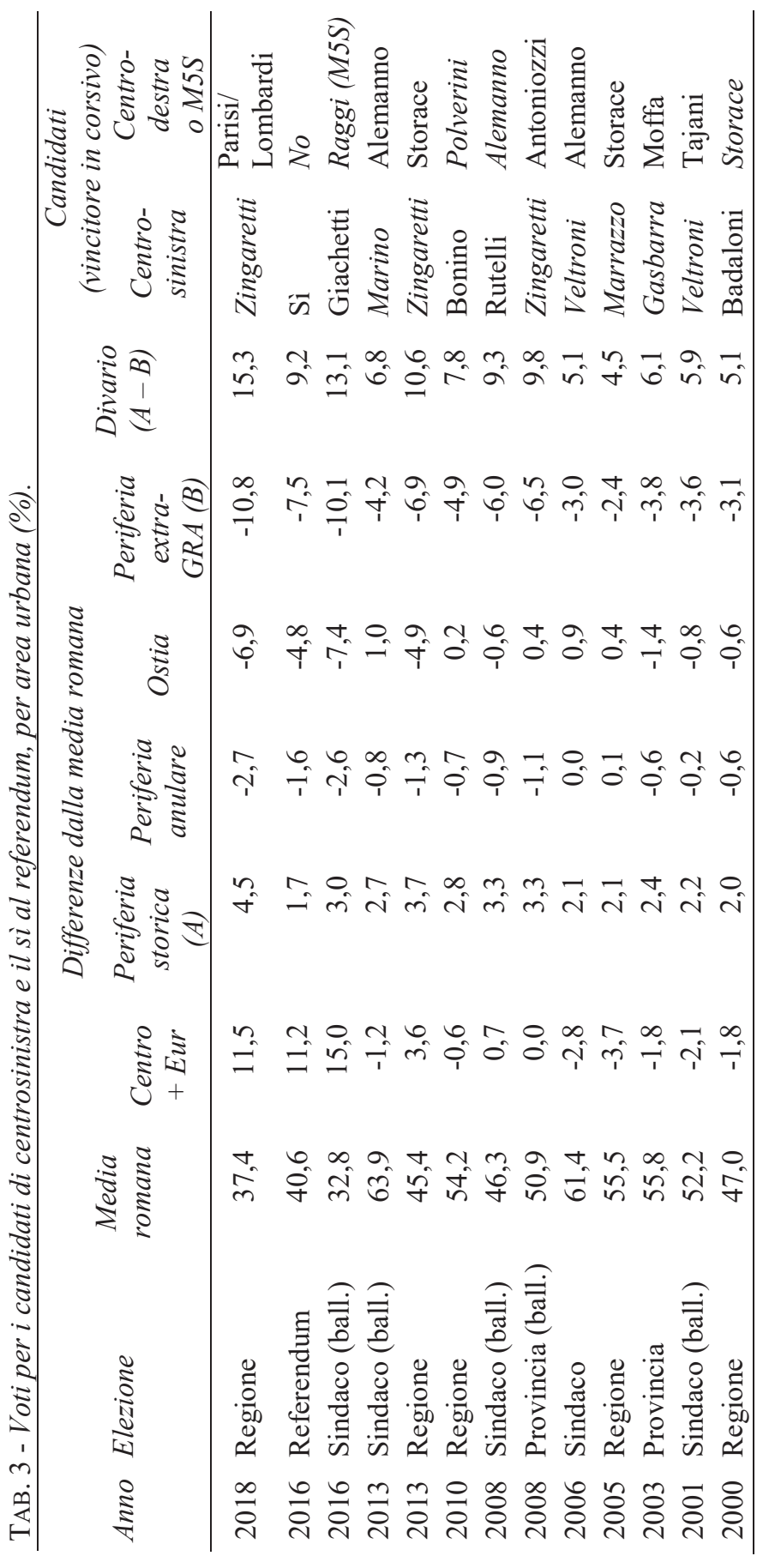


Dal 2000 sono sempre elevati i divari per i candidati di centrosinistra tra la periferia storica e quella più esterna, che vanno dai 4,5 punti percentuali del 2005 ai 15,3 del 2018, e anzi dal 2008 il gap rimane quasi sempre sopra 9. Alcune elezioni tuttavia mostrano caratteri peculiari, poiché erano distribuiti più uniformemente i voti sia per Badaloni e Marrazzo alla Regione nel 2000 e nel 2005, sia per il secondo mandato da sindaco di Veltroni nel 2006, tre candidati le cui caratteristiche personali hanno superato le tradizionali divisioni politiche, essendo i primi due popolari giornalisti e l'ultimo un celebrato sindaco con consenso bipartisan.

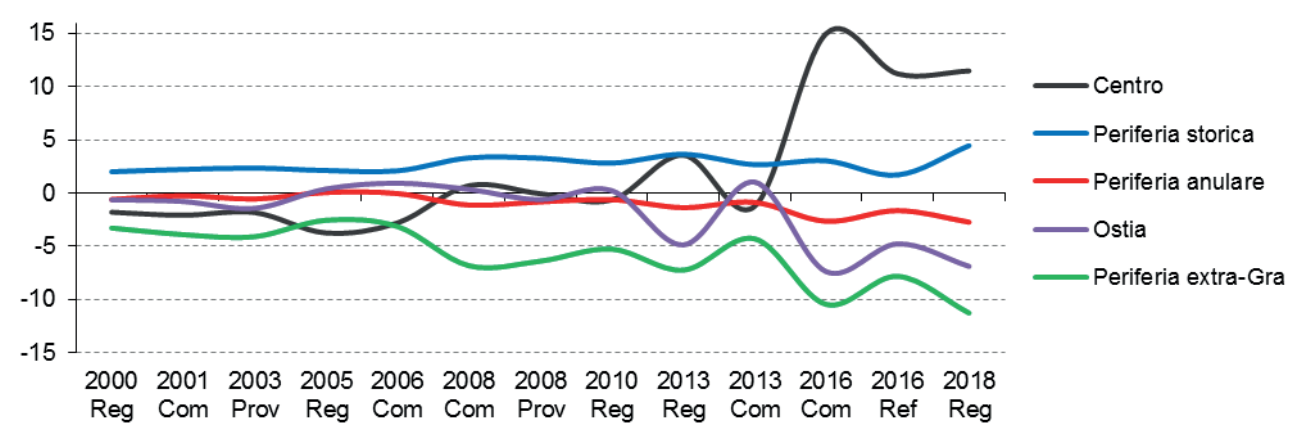

FIG. 4 - Differenze dei voti per $i$ candidati del centrosinistra rispetto alla media romana, per fascia urbana (punti \%).

Solo i risultati del 2016 e 2018 sono stati significativamente difformi rispetto alle elezioni precedenti, segnando una discontinuità nelle dinamiche tra il centro, le periferie e Ostia, pur confermando e anzi rafforzando la legge gravitazionale secondo l'attrazione del Campidoglio. Infatti il centro è diventata l'area urbana dove si registra ormai il maggiore consenso per la coalizione di centrosinistra, a livelli notevoli e inconsueti, fino a 15 punti percentuali in più rispetto alla media romana, superando nettamente la periferia storica composta dai quartieri popolari e tradizionalmente di sinistra. Al contempo il risultato del centrosinistra è peggiore rispetto al trend nella periferia anulare interna al GRA, in quella più esterna (in quest'ultima circa 10 punti percentuali in meno rispetto alla media romana) e sul litorale di Ostia.

La stessa tendenza continua anche nei comuni limitrofi a Roma, alcuni dei quali cresciuti notevolmente come popolazione negli ultimi anni, almeno in parte a causa dei romani trasferiti in cerca di case a costi ragionevoli. La prima cintura dell'hinterland ha dato alle politiche del 2013 e del 2018 un grande successo al M5S (eccetto che nella Valle dell'Aniene e 
in parte dei Castelli), e l'elezione negli anni recenti di vari sindaci tra cui Pomezia, Civitavecchia, Ardea, Nettuno, Genzano, Marino e Anguillara.

\section{Le elezioni politiche 2018}

Le elezioni politiche hanno in gran parte confermato le dinamiche del voto alle comunali del 2016, che a loro volta - come detto - avevano mostrato una geografia politica un po' diversa rispetto agli anni precedenti (cfr. Fig. 5 e Fig. 6).

Il voto per la coalizione di centrosinistra (formata da PD, +Europa, Insieme e Civica Popolare), che in totale ha ottenuto 324 mila voti pari al $28 \%$, è nettamente decrescente allontanandosi dal centro della città, poiché prevale solo nelle zone più centrali $(40 \%)$ e, diversamente dal 2016, ma di pochissimo, nella periferia storica (quasi $31 \%$, peraltro egemonizzata dal centrosinistra fino al 2013). Dinamiche simili valgono per LeU, con 68mila voti pari al $4,6 \%$, che salgono sopra il $5 \%$ nel centro e nella periferia storica e scendono sotto il 3\% fuori dal GRA. A ciò corrisponde una tendenza opposta per il M5S, che ha ottenuto 446mila voti pari a meno del $31 \%$, e che cresce man mano che ci si allontana verso i confini comunali, prevalendo nella periferia anulare meno densa (33\%) e soprattutto a Ostia $(38 \%)$ e negli insediamenti sparsi fuori dal GRA (39\%), sebbene abbia perso alcuni punti percentuali rispetto al 2016, probabilmente per i problemi dell'Amministrazione comunale guidata dalla Sindaca Raggi. Questo calo è andato a vantaggio della Lega, che per la prima volta si afferma con percentuali notevoli nelle periferie romane, e che mostra un andamento dei voti simile a quello del M5S, con il massimo fuori dal GRA. Nel complesso della città, è infatti proprio la coalizione di centrodestra (composta da Forza Italia, Lega, Fratelli d'Italia e Noi con l'Italia) a vincere con 454 mila voti pari al $31 \%$, con un andamento più omogeneo rispetto agli altri due poli, caratterizzato comunque da un maggiore consenso fuori dal GRA (34\%) grazie a Forza Italia e Lega, e con un forte incremento rispetto alle precedenti elezioni che gli ha permesso di avere un vantaggio di mezzo punto percentuale sul M5S e di circa tre punti sul centrosinistra. 


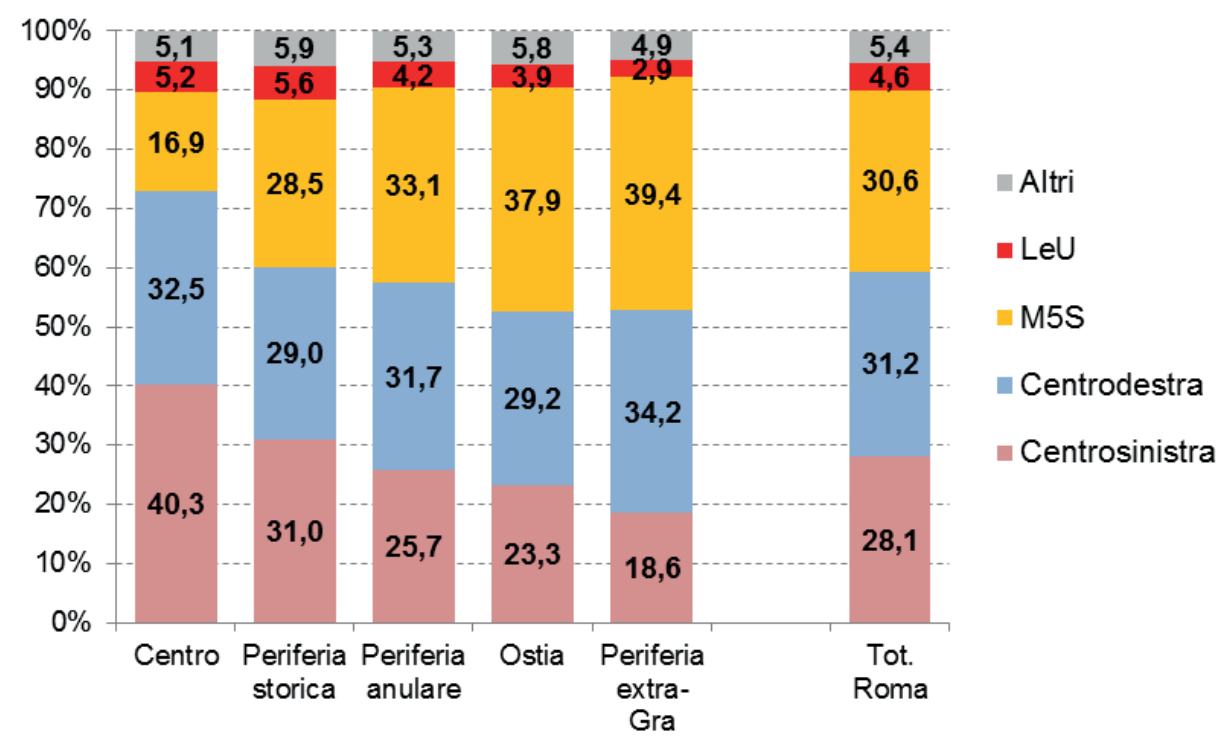

FIG. 5 - Voti per candidati uninominali alla Camera 2018, per fascia urbana (\%).

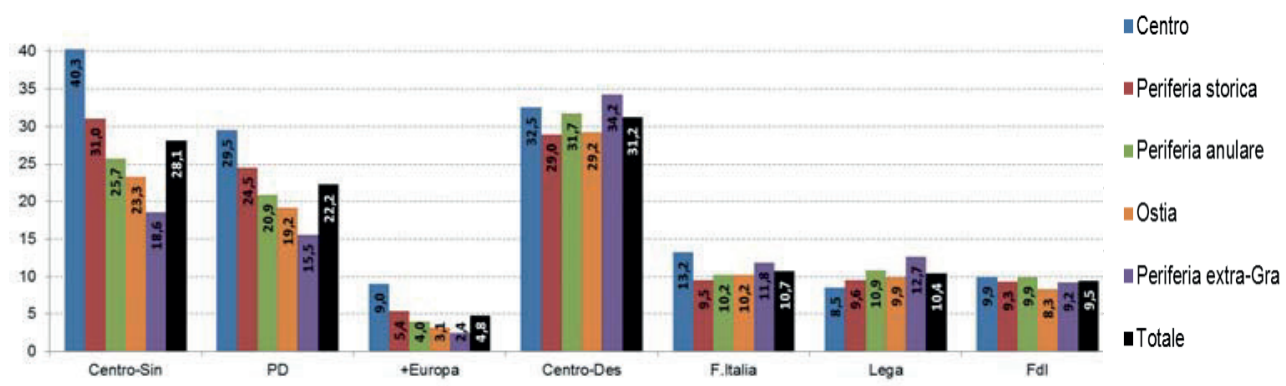

FIG. 6 - Voti per coalizioni e liste alla Camera 2018, per fascia urbana (\%).

Se calcoliamo la differenza dei voti alla Camera uninominale 2018 rispetto al primo turno del Comune 2016 (cfr. Fig. 7), osserviamo come il M5S perde tra 4 e 7 punti percentuali nel centro, nella periferia storica e in quella anulare, mentre contiene il calo in soli 2 punti nella periferia esterna al GRA. Al contrario, la coalizione di centrosinistra guadagna 6,5 punti percentuali nel centro e circa 3 sia nella periferia storica che in quella anulare, ma partendo dal dato molto deludente di Giachetti nel 2016, anche nelle zone di tradizionale insediamento della sinistra. Infine, l'altra coalizione di centrodestra rispetto alla somma dei voti per Meloni e Marchini, nonché LeU rispetto a Fassina, mantengono sostanzialmente le stesse percentuali. 


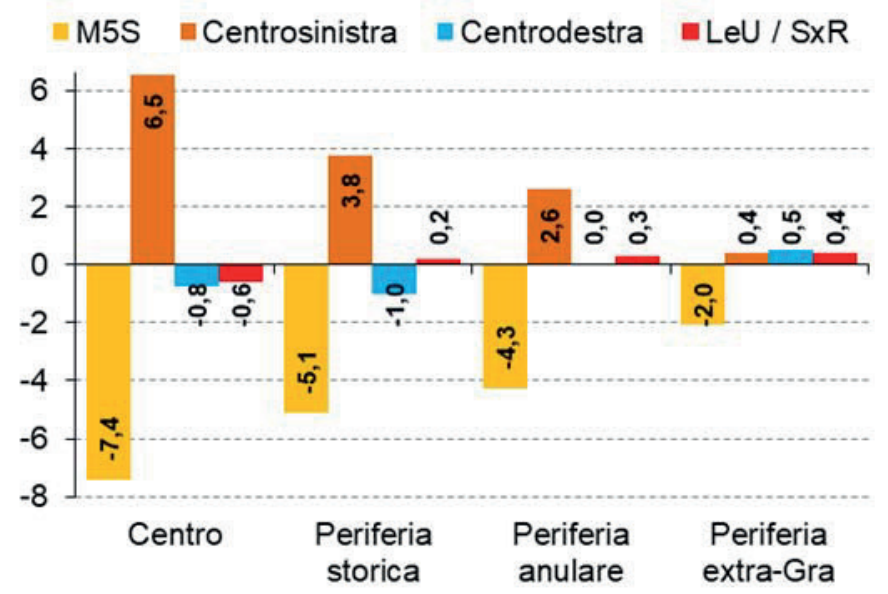

FIG. 7 - Differenza dei voti alla Camera uninominale 2018 rispetto al primo turno del Comune 2016, per fascia urbana (punti \%).

Andando più nel dettaglio, al livello delle zone urbanistiche, rappresentiamo in mappa $i$ voti in percentuale per i candidati uninominali di centrosinistra, centrodestra, M5S e LeU (cfr. Fig. 8 e Fig. 9).

Il centrosinistra (mappa in alto a sinistra) mostra le percentuali maggiori nei Municipi I e II, dove erano candidati Gentiloni e Madia, nonché nel resto dell'area all'interno o subito fuori dall'anello ferroviario, oltre all'Eur; in particolare, le zone urbanistiche migliori sono tutte centrali: Trastevere $(48 \%$, dove anche il PD ottiene il massimo con oltre il 34\%), Celio (46\%, dove invece è +Europa a ottenere il massimo col 12\%), Aventino, Della Vittoria, Flaminio, Centro Storico e Salario (43-45\%). Al contrario, il centrosinistra scende sotto al $20 \%$ quasi ovunque fuori dal GRA, soprattutto nei quadranti est del VI Municipio a San Vittorino, Borghesiana, Tor Cervara, Lunghezza, Acqua Vergine e Torre Angela (13-16\%) e ovest a Pantano di Grano, Ponte Galeria, Boccea e Santa Maria di Galeria (circa 15\%).

Il centrodestra (mappa in alto a destra) ha avuto il consenso maggiore sia nelle tradizionali roccaforti "nere" di Roma Nord (II e XV Municipio), sia nelle periferie fuori dal GRA dei quadranti nord-ovest ed est (tutto il VI Municipio e le parti esterne del XII, XIII e XIV), oltre alle ville dell'Appia Antica (VIII Municipio): tra le prime Parioli (45\%, dove anche Forza Italia ottiene il massimo con quasi il 23\%), Tor di Quinto, Acquatraversa, Grottarossa Ovest (dove Fratelli d'Italia registra il massimo con quasi il 15\%) e Farnesina (42-44\%); tra le seconde a ovest Boccea, La Storta, Cesano, Pantano di Grano, Santa Cornelia, Santa Maria di Galeria e Prima Porta (40-43\%), a est Borghesiana e San Vittorino (38\%, dove è la 
Lega a raggiungere il massimo con il 15-16\%). Il minimo per il centrodestra si registra in alcuni quartieri centrali e nella periferia storica: San Lorenzo (21\%), Testaccio (22\%), Trastevere (23\%), Montesacro, Garbatella, Tiburtino Sud, Celio, Ostiense e Gianicolense (24-26\%), oltre a Malafede, fuori dal GRA nel X Municipio.

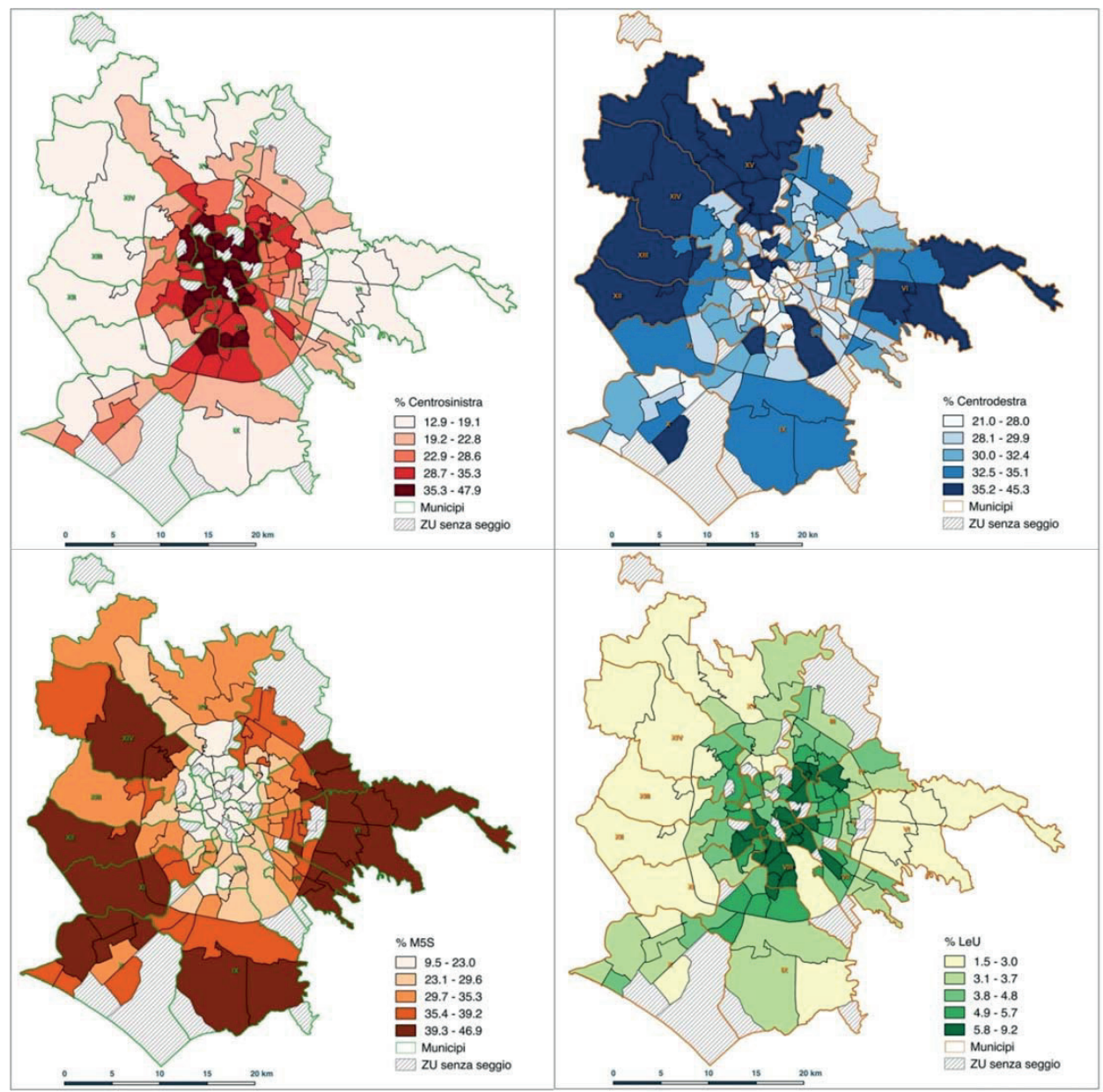

FIG. 8 - Voti per la Camera uninominale 2018, per zone urbanistiche (\%). 

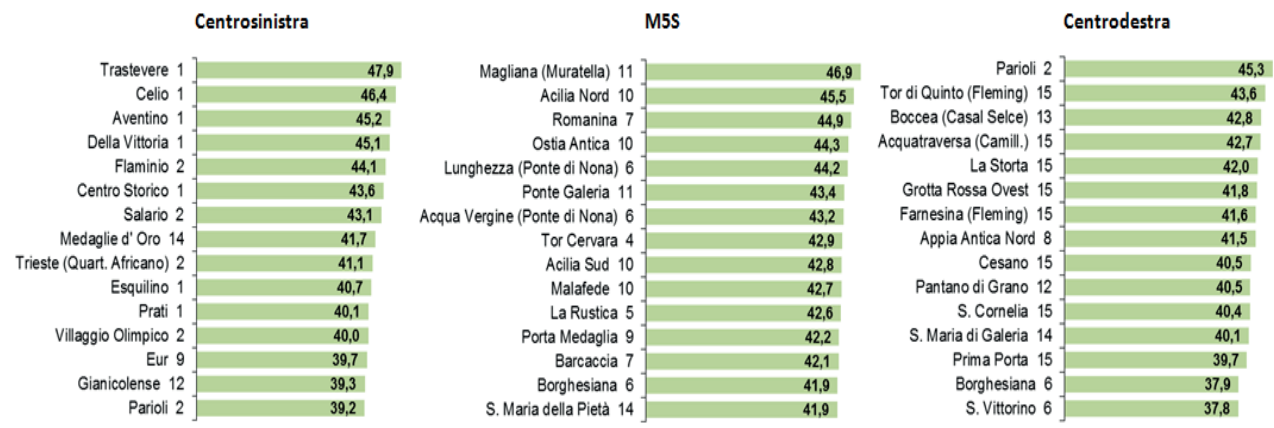

FIG. 9 - Migliori risultati delle coalizioni nelle zone urbanistiche per la Camera uninominale $2018(\%)$.

Per il M5S (mappa in basso a sinistra) vale l'ormai consueta prevalenza a ridosso o fuori dal GRA, in tutti i quadranti ma in particolare nelle periferie a sud-ovest nel X e XI Municipio verso il litorale e ad est nel VI Municipio e in quelli limitrofi: tra le prime Magliana (47\%), Acilia Nord (45,5\%), Ostia Antica, Ponte Galeria, Acilia Sud e Malafede (43-44\%); tra le seconde Romanina (45\%), Lunghezza e Acqua Vergine (che corrispondono all'area di Ponte di Nona, 43-44\%), Tor Cervara e La Rustica (quasi 43\%). Al contrario, le percentuali più basse sono state registrate in tutti i quartieri centrali e semiperiferici, soprattutto nel quadrante nord: il minimo a Parioli (9,5\%), e poi Centro Storico, Salario e Farnesina (circa 13\%), Celio, Tor di Quinto, Medaglie d'Oro, Aventino, Prati, Eur e Della Vittoria (15-16\%). Rispetto alle comunali 2016, il M5S ha perso consensi un po' ovunque, probabilmente per le difficoltà dell'Amministrazione comunale guidata dalla sindaca Raggi, ma se il calo è stato più consistente nelle zone centrali (-7 punti percentuali), dove il movimento aveva preso meno voti, appare invece più contenuto nella periferia storica $(-5)$ e anulare $(-4)$, e soprattutto nelle sue roccaforti fuori dal GRA (solo -2,4).

Infine, LeU (mappa in basso a destra) ha ottenuto voti soprattutto nelle zone centrali e nella periferia storica, in maniera simile al centrosinistra, differenziandosene però per una minore concentrazione del consenso nei quartieri a nord-ovest e maggiore in quelli a sud-est nei Municipi VII e VIII, sempre comunque all'interno del GRA. Le zone urbanistiche con le migliori percentuali per LeU sono infatti San Lorenzo $(9,2 \%)$, Montesacro (8,3\%), Ostiense e Garbatella (7,6\%), Testaccio, Appio, Celio, Tuscolano, Gianicolense e Latino (circa 7\%). I quartieri peggiori sono invece tutti fuori o a ridosso del GRA nei quadranti nord-ovest (Santa 
Maria di Galeria 1,5\%) ed est soprattutto nel VI Municipio (San Vittorino $1,9 \%)$.

All'interno delle coalizioni (cfr. Fig. 10), le liste del centrosinistra sono abbastanza sovrapponibili, soprattutto per quanto riguarda PD e +Europa, le cui distribuzioni del voto nelle zone urbanistiche hanno un indice di correlazione positivo e significativo $(0,90)$, ed è peraltro elevata anche tra PD e LeU $(0,70)$. Invece i partiti del centrodestra appaiono perfettamente complementari, poiché Forza Italia e Fratelli d'Italia hanno le percentuali più elevate nei quartieri benestanti di Roma Nord, mentre la Lega mostra invece una forte capacità di attrazione nelle zone periferiche con il maggiore disagio socio-economico, e soprattutto a est nel VI Municipio, con un indice di correlazione tra Forza Italia e Lega positivo ma basso $(0,33)$, come anche tra Forza Italia e Fratelli d'Italia $(0,34)$.
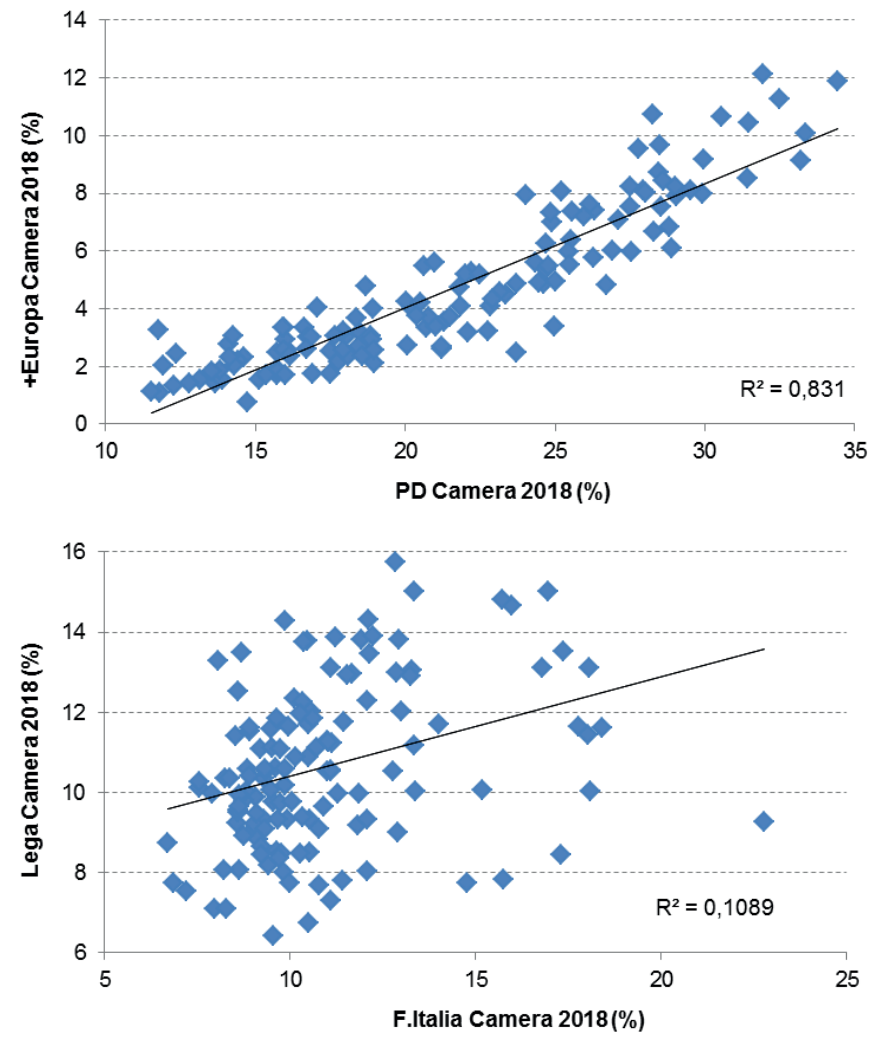

FIG. 10 - Correlazione dei voti nelle zone urbanistiche per la Camera uninominale 2018 (\%). 


\section{Le elezioni regionali 2018}

Alle elezioni regionali, l'esito più interessante è la riconferma di Zingaretti (sostenuto da PD, LeU, +Europa, Insieme, Centro Solidale e Lista Civica) alla presidenza della Regione, nonostante le liste di centrosinistra abbiano avuto meno voti rispetto al centrodestra, e nello stesso giorno in cui la sua coalizione usciva sconfitta dalle elezioni politiche. È stata quindi un'ottima performance personale del governatore uscente (Passarelli e Valbruzzi, 2018), un vero e proprio "effetto Zingaretti" (Carrieri, 2018), probabilmente favorito dal fatto che la coalizione era larga ma credibile, comprendendo anche LeU diversamente dalle elezioni politiche, e che al contrario il centrodestra si presentava diviso tra i due candidati Parisi (appoggiato da Forza Italia, Lega, Fratelli d'Italia, Noi con l'Italia ed Energie per l'Italia) e Pirozzi, mentre Lombardi del M5S arrivava terza.

A Roma il centrosinistra vince con oltre il $37 \%$ dei voti, circa 10 punti percentuali in più rispetto a centrodestra (28\%) e M5S (26\%), ma rimangono comunque in gran parte costanti le dinamiche del voto della Camera: l'andamento dei consensi per Zingaretti è infatti nettamente decrescente allontanandosi dal centro della città (cfr. Fig. 11). Tuttavia, a differenza della Camera, il candidato del centrosinistra prevale non solo nelle zone più centrali $(49 \%)$ e nella periferia storica intorno all'anello ferroviario (42\%), dove il distacco da Parisi e Lombardi è ampio, ma anche con uno scarto più ridotto nella periferia anulare interna al GRA (quasi $35 \%$ ). Di conseguenza, la vittoria di Zingaretti nel Lazio, che è dipesa da un margine di soli 55 mila voti in tutta la regione rispetto a Parisi, si basa in primo luogo sulla parte di Roma dentro al GRA, dove ha ottenuto 157mila voti più di Parisi e 184 mila più di Lombardi. Nella periferia fuori dal GRA continua invece a prevalere il M5S (quasi 35\%), ma con un margine limitato rispetto al centrodestra (30,5\%), analogamente alle elezioni politiche. Anche in questo caso la coalizione di centrodestra mostra dinamiche più omogenee rispetto agli altri due poli, sebbene comunque caratterizzate da un maggiore consenso fuori dal GRA. 


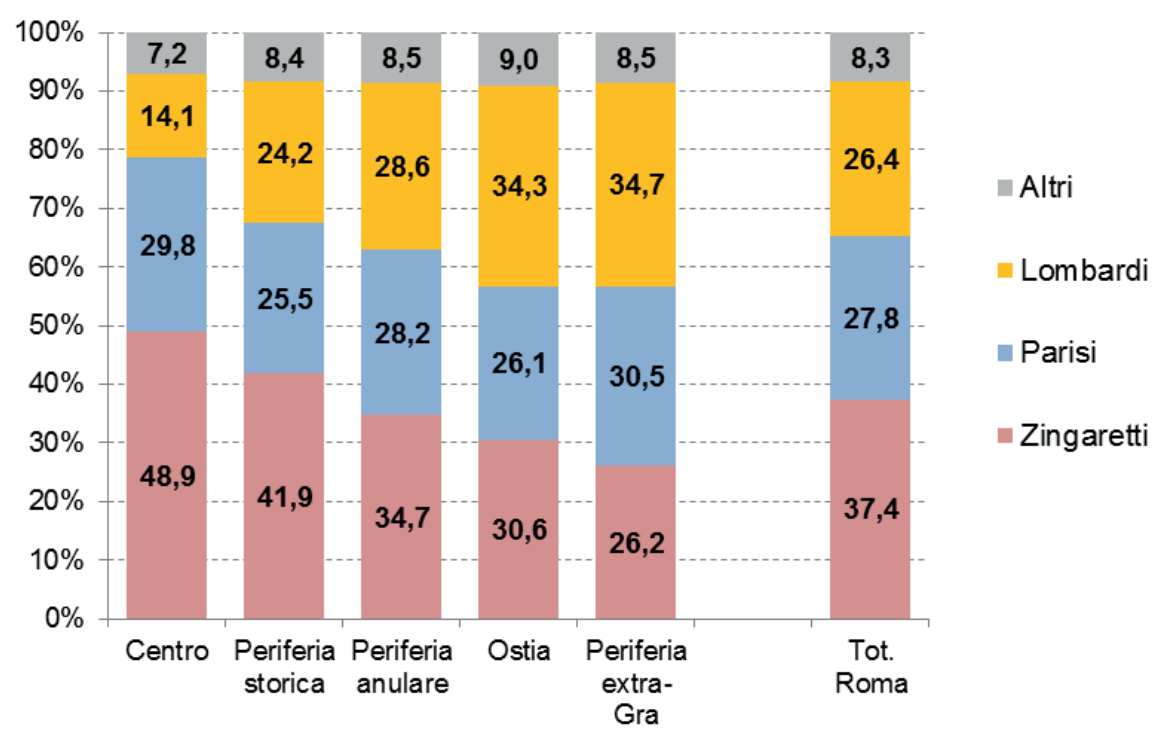

FIG. 11 - Voti per i candidati presidenti della Regione 2018, per fascia urbana (\%).

Tuttavia, sia il centrodestra che il M5S ottengono alle elezioni regionali circa 4-5 punti percentuali in meno rispetto alle politiche, a tutto vantaggio appunto di Zingaretti, che ottiene 4,7 punti in più rispetto alla somma del risultato del centrosinistra e di LeU nei collegi uninominali della Camera (cfr. Fig. 12).

a)

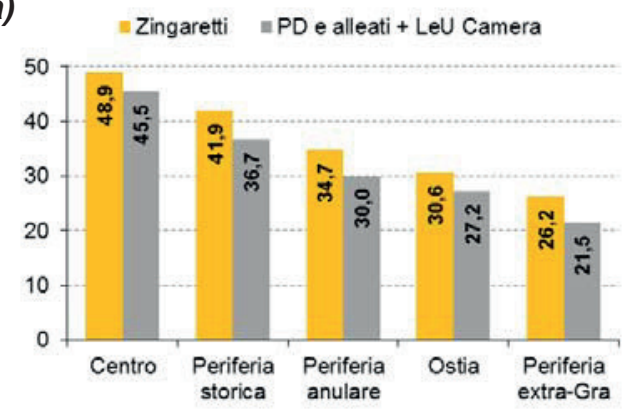

b)

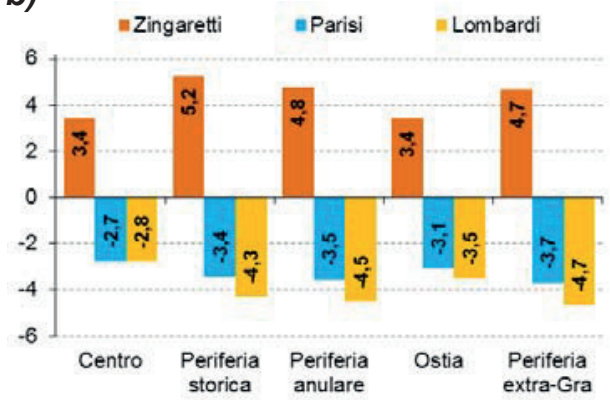

FIG. 12 - a) Voti per Zingaretti e per $i$ candidati uninominali di PD e alleati + LeU, per fascia urbana (\%);

b) Differenza dei voti per i candidati alla Regione rispetto alle coalizioni alla Camera, per fascia urbana (punti \%). 


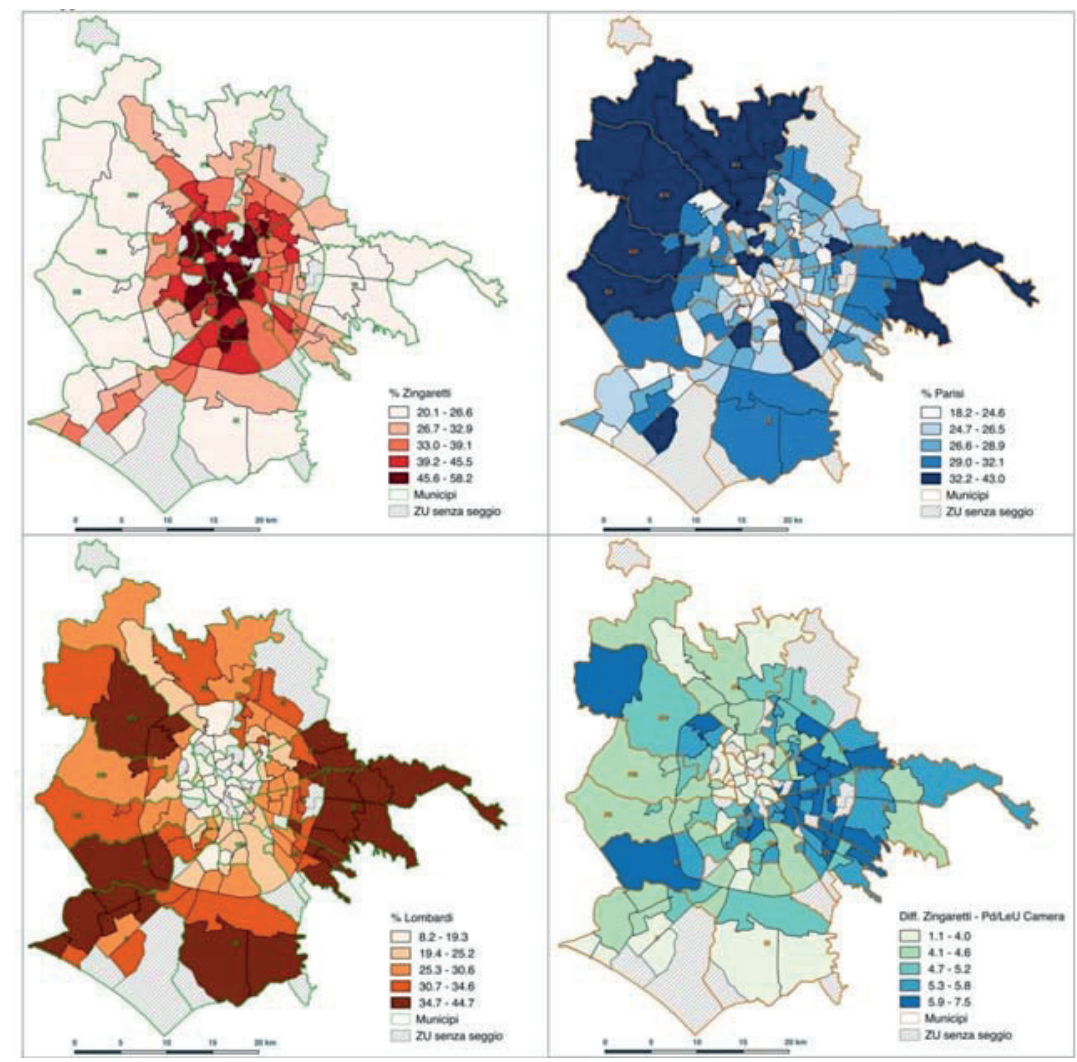

FIG. 13 - Voti per i candidati presidenti della Regione 2018, per zone urbanistiche (\%).

Il dettaglio delle zone urbanistiche nelle mappe è molto simile rispetto alle politiche, con $i$ voti in percentuale per $i$ candidati uninominali di centrosinistra, centrodestra e M5S (cfr. Fig. 13). È però interessante mostrare una sorta di quantificazione dell'"effetto Zingaretti" (mappa in basso a destra) come differenza tra $i$ voti per il candidato presidente (543mila) e la somma di centrosinistra e LeU nei collegi uninominali della Camera (477mila). Questa differenza è sempre positiva in ogni zona urbanistica, e varia tra un massimo di 7,5 punti percentuali a San Lorenzo e Testaccio e un minimo di 1,1 punti a Celio, Prati e Centro Storico. Nonostante che il massimo e il minimo siano registrati entrambi in quartieri centrali, l'effetto del candidato è maggiore in tutto il quadrante est, nonché nella periferia storica a sud e in alcune zone extra GRA a ovest. Le differenze sono invece minori in tutte le zone centrali dove già il centrosinistra e LeU avevano vinto, e quindi Zingaretti aveva un margine di crescita inferiore. 


\section{Fattori demografici, sociali, economici e urbanistici}

L'analisi del voto secondo la vicinanza o meno dal centro della città è ovviamente una semplificazione, in quanto il consenso elettorale dipende dalle diverse caratteristiche demografiche, sociali, economiche e urbanistiche dei quartieri. Per approfondire questi fattori vediamo l'andamento del voto per i tre poli alle elezioni politiche e per Zingaretti alle regionali, suddividendo le zone urbanistiche in tre gruppi di uguale numerosità, secondo il livello alto, medio o basso dei vari indicatori presi in esame (cfr. Fig. 14). Per dare evidenza dell'articolazione territoriale delle disuguaglianze socio-economiche prendiamo a riferimento l'indice di disagio $^{3}$, che risulta più elevato nelle zone urbanistiche periferiche e in gran parte del quadrante orientale della città (cfr. Fig. 15).

Il M5S ottiene più voti dove la densità di popolazione è bassa $(36 \%)$, l'incremento dei residenti è alto $(40 \%)$, l'età media è bassa $(39 \%)$, i componenti del nucleo familiari sono molti (39\%), i laureati sono pochi (ancora 39\%), il tasso di occupazione è basso (36\%), il tasso di disoccupazione è alto $(38 \%)$, la disponibilità di piazze per ettaro, come indicatore di capitale sociale, è scarsa $(39 \%)$, il disagio socio-economico è alto $(37,5 \%)$. Al contrario, il centrosinistra e Zingaretti hanno più voti dove la densità di popolazione è alta (rispettivamente 32 e $42 \%$ ), la crescita dei residenti è bassa o negativa (ancora 32 e 42\%), l'età media è alta (34 e quasi $45 \%$ ), i componenti del nucleo familiari sono pochi (oltre 36 e quasi $47 \%$ ), i laureati sono molti ( 37 e 46\%), il tasso di occupazione è alto (quasi 36 e oltre $45 \%$ ), il tasso di disoccupazione è basso (oltre 35 e quasi $45 \%$ ), la disponibilità di piazze per ettaro è alta (34 e 44\%), il disagio socioeconomico è basso (35 e 44\%). Il centrodestra mostra invece un consenso abbastanza omogeneo, con differenze limitate, eccetto le variabili urbanistiche e l'età media, ma comunque inferiori a 5 punti percentuali, proprio grazie alle caratteristiche interclassiste dovute alla complementarietà nel consenso elettorale tra Forza Italia e Lega.

Analisi statistiche di tipo sia esplorativo sia inferenziale confermano un impatto positivo sul voto al centrosinistra per l'anzianità della popolazione, la disponibilità di beni comuni e relazionali (di cui sono proxy la densità di popolazione e di piazze) e - solo nelle elezioni 2016 e 2018 - lo

\footnotetext{
${ }^{3}$ Indice calcolato da Roma Capitale sulla base di disoccupazione, occupazione, concentrazione giovanile (popolazione con meno di 25 anni) e scolarizzazione (diploma superiore o laurea) www.comune.roma.it/webresources/cms/documents/Gli_indici_di_disagio_sociale_ed_edilizio_a _Roma_W.pdf.
} 
status socio-economico e la centralità o attrattività urbana, mentre al contrario l'esclusione dalle opportunità educative e lavorative ha un effetto negativo (Tomassi, 2013, pp. 432-439; 2018).

a)
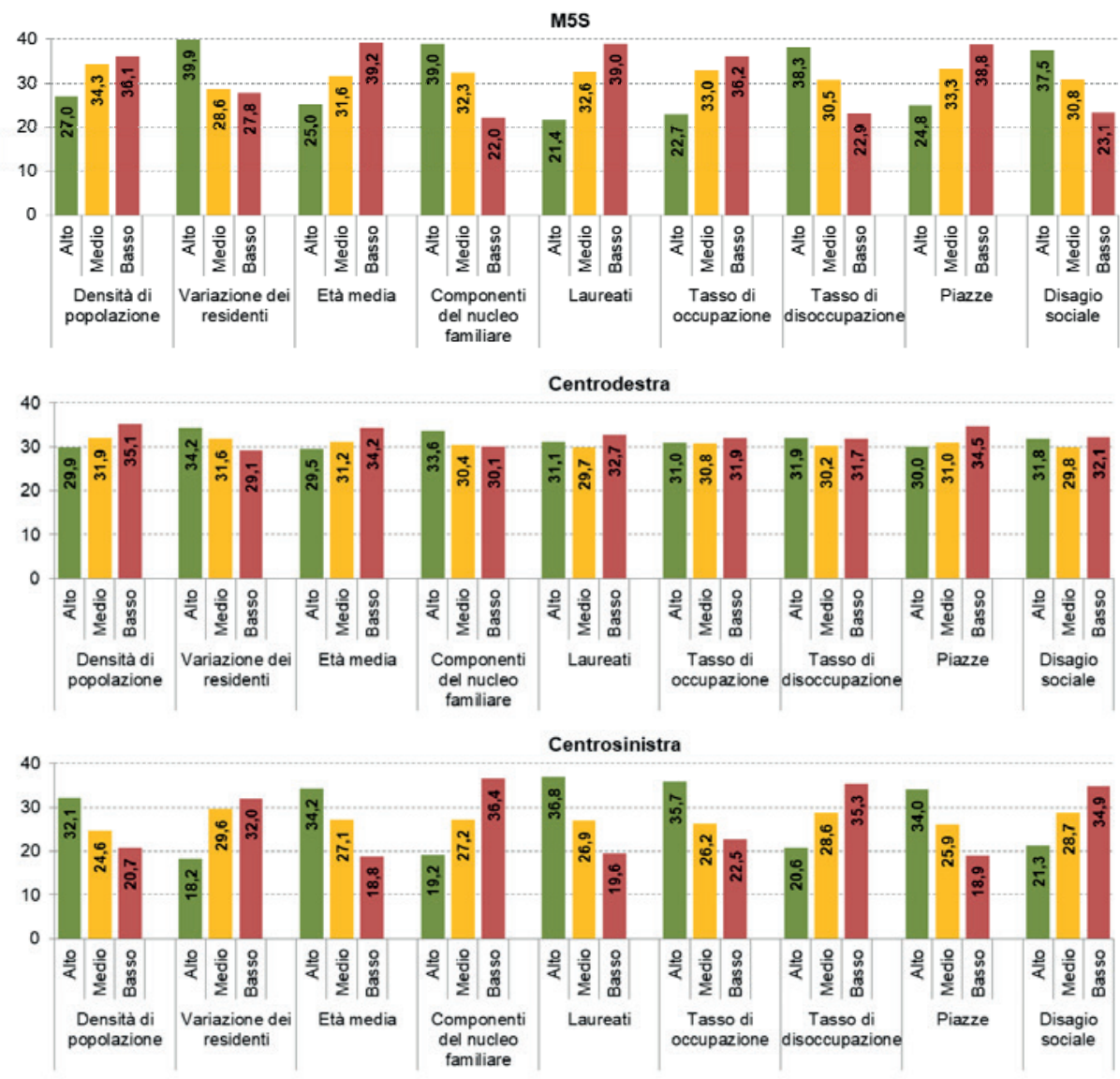

b)

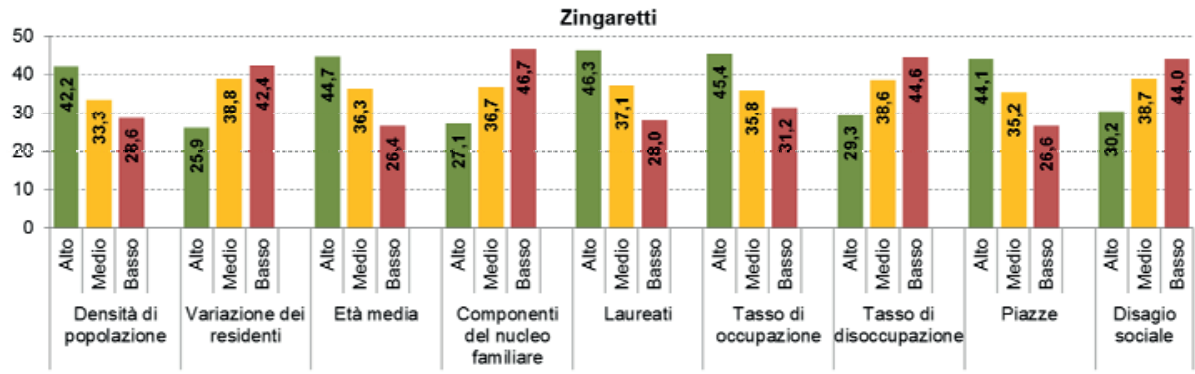

FIG. 14 - Voti a) alla Camera uninominale e b) alla Regione 2018, per caratteristiche delle zone urbanistiche (\%). 


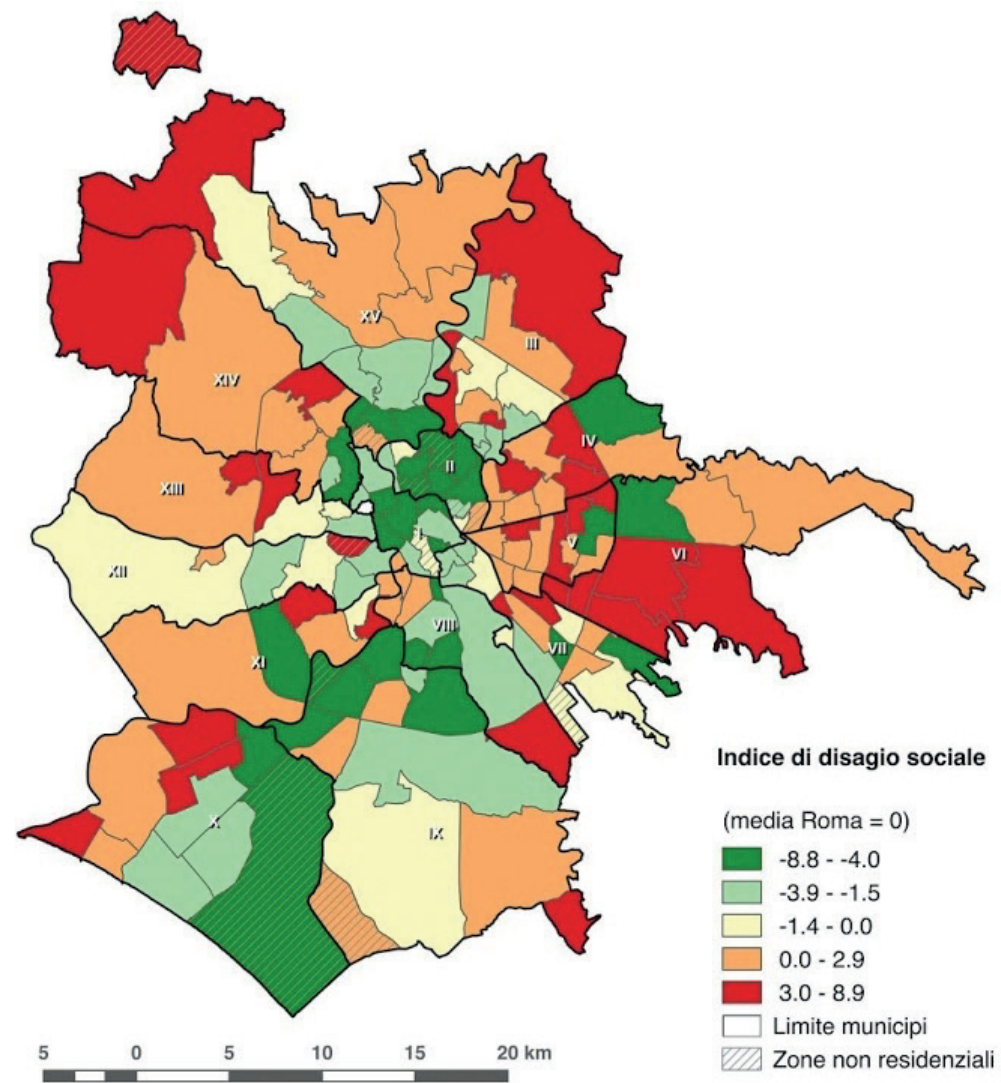

FIG. 15 - Indice di disagio sociale 2011, per zona urbanistica (media Roma =0).

\section{Spunti conclusivi}

Il M5S prevale negli insediamenti in rapida espansione vicini o esterni al GRA, isolati dal tessuto urbanistico della città consolidata, con limitate opportunità in termini di istruzione, occupazione, offerta di servizi e spazi pubblici e accessibilità dei trasporti, dove di conseguenza l'indice di sviluppo umano rimane sotto la media romana e al contrario il disagio sociale è superiore alla media. Ciò vale in parte anche per il centrodestra, che conserva comunque le tradizionali roccaforti "nere" a nord della città, soprattutto grazie alla rapida crescita elettorale della Lega nelle periferie. L'opposto vale per il centrosinistra, che ottiene i maggiori consensi dove la città è consolidata, l'offerta di servizi pubblici e privati è più consistente, le opportunità di incontro sono maggiori e i residenti subiscono meno l'impatto della crisi economica e delle disuguaglianze, cosicché l'indice di 
sviluppo umano risulta superiore rispetto alla media e il disagio sociale è inferiore.

È proprio nei quartieri intensivi e popolari della periferia storica che, almeno in parte, persiste l'eredità politico-culturale della sinistra romana che qui aveva una forte egemonia elettorale, sebbene anche in questa fascia le migliori condizioni socio-economiche non compensano altri elementi di insoddisfazione relativi al governo nazionale o alle dinamiche globali. In generale, nelle metropoli, i quartieri densi permettono relazioni sociali più ravvicinate e ricche, nonché spazi pubblici e collettivi più facilmente accessibili, e quindi facilitano politiche inclusive e cosmopolite (Sellers et al., 2013). Il netto divario territoriale del 2016 e 2018 tra le aree centrali che votano il centrosinistra e il resto della città, dove vince con ampio margine il M5S, evidenzia come l'elettorato del PD (ma anche delle liste alla sua sinistra) sia sempre più fortemente caratterizzato da alti livelli di istruzione $\mathrm{e}$ reddito, mentre è scarsa la capacità attrattiva in zone periferiche con maggiore disagio socio-economico.

Un profilo interclassista rimane solo per il centrodestra, i cui partiti appaiono perfettamente complementari, poiché Forza Italia e Fratelli d'Italia hanno le percentuali più elevate nei quartieri benestanti di Roma Nord, mentre la Lega mostra una grande capacità di attrazione nelle zone periferiche con il maggiore disagio socio-economico, e soprattutto a est nel VI Municipio. È infatti negli insediamenti sparsi della periferia anulare ed esterna al GRA che penetrano più facilmente i messaggi politici populisti, se non persino nazionalisti e xenofobi (Rossi, 2018). Nei casi più estremi, alcuni quartieri periferici dove si sommano problemi e criticità di varia natura hanno vissuto vere e proprie rivolte urbane contro gruppi deboli e minoritari, fomentate da politici locali di destra e movimenti neofascisti, come a Tor Sapienza (Bianchi, 2014; Selmini, 2016).

Esiste certamente una tendenza generale alla perdita di consenso per le istanze progressiste tra le fasce sociali marginali più deboli, meno protette e maggiormente colpite dalla crisi economica, non solo a Roma e non solo in Italia: le analisi a livello nazionale mostrano il centrosinistra confinato nei centri urbani (Maccagno, 2018) e una maggiore propensione al voto per il PD nelle classi medio-alte (De Sio, 2018), cosicché sembra essere stata la condizione socio-economica e la marginalità sociale a decidere le elezioni (De Benedetto e De Paola, 2018). In un quadro politico - sia nazionale che romano - sempre più frammentato, fluido, personalizzato e instabile, la volontà di rompere con $\mathrm{i}$ vecchi partiti si manifesta più forte nelle zone periferiche dove lo sviluppo urbanistico non è accompagnato da servizi 
adeguati e la stagnazione economica ha fatto sentire più forte la crisi dei redditi familiari (Diletti e Gritti, 2016).

Ma al di là di questo, bisognerebbe chiedersi perché le caratteristiche delle periferie romane più estreme sfavoriscono i candidati del centrosinistra almeno dal 2000, ben prima della crisi e dell'ondata populista. Al riguardo, due ordini di ragionamento inducono a pensare che lo spostamento di residenti verso le periferie più lontane crei maggiori difficoltà ai partiti del centrosinistra rispetto al centrodestra e ai populisti. Il primo motivo, specificatamente romano, è l'identificazione del centrosinistra con il governo della città, nel ventennio di quasi ininterrotta amministrazione comunale fino al 2015, tranne la parentesi di Alemanno dal 2008 al 2013, e di gran parte dei presidenti dei Municipi. Tuttavia, l'effettiva crescita economica di questo periodo contrasta con le crescenti polarizzazioni dal punto di vista distributivo, sociale e urbanistico, e con le difficoltà anche della normale amministraazione quotidiana in un territorio comunale vasto come quello di Roma, che incide soprattutto nei quartieri periferici più lontani dal Campidoglio, facendo sì che la responsabilità - a torto o a ragione - sia ascritta al centrosinistra che ha governato a lungo Comune e Municipi. La seconda ragione, che accomuna tutte le periferie delle grandi città italiane, riguarda le forme dell'attività politica su un territorio complicato e spesso privo di luoghi di incontro, dove il centrosinistra ha subito da un lato prima la presenza mediatica del centrodestra e poi l'attivismo su internet del M5S, e dall'altro lato la deriva verso forme di partito notabilare "in franchising", destrutturato rispetto ai vecchi partiti di massa (Tocci, 2015, pp. 15-26), talvolta dirottato verso il potere fine a se stesso (Barca, 2015), i cui leader locali conquistano consenso personale controllando i voti di preferenza (Marchianò, 2015). 


\section{Riferimenti bibliografici}

ACCIARI P. e MocetTI S. [2013], «Una mappa della disuguaglianza del reddito in Italia», Banca d'Italia - Questioni di Economia e Finanza, 208.

AlleVA G. [2017], Audizione alla Commissione parlamentare di inchiesta sulle condizioni di sicurezza e sullo stato di degrado delle città e delle loro periferie, Roma, Camera dei Deputati.

BARCA F. (cur.) [2015], Mappa il PD di Roma. Relazione finale, rapporto di Luoghi Idea(li) per il PD di Roma.

Bianchi L. [2014], Come $i$ neofascisti provano a prendersi le periferie romane, Internazionale, pubblicato on-line.

CAMAGNi R. [2016], Metropolitan challenges and strategies in the years to come, International conference «Greater Paris and Metropolitan Strategies in Europe», Parigi.

CARRIERI L. [2018], Regionali nel Lazio: l'effetto Zingaretti e le divisioni del centrodestra, CISE-LUISS, pubblicato on-line.

CAUSI M. [2018], SOS Roma. La crisi della capitale. Da dove viene, come uscirne, Roma, Armando.

Cipollini R. e Truglia F.G. [2015], La metropoli ineguale. Analisi sociologica del quadrante Est di Roma, Ariccia, Aracne.

COMMISSIONE PARLAMENTARE DI INCHIESTA SULLE CONDIZIONI DI SICUREZZA E SULLO STATO DI DEGRADO DELLE CITTÀ E DELLE LORO PERIFERIE [2017], Relazione sull'attività svolta dalla Commissione, Roma, Camera dei Deputati.

De Benedetto M.A. e De PaOla M. [2018], Se la condizione socioeconomica decide il voto, LaVoce.info, pubblicato on-line.

De Muro P. [2013], «Disuguaglianze capitali», in Italianieuropei, 2/3.

De Muro P., Monni S. e Tridico P. [2011], «Knowledge-based economy and social exclusion: shadows and lights in the Roman socioeconomic model», in International Journal of Urban and Regional Research, 35, 6, pp. 1212-1238.

De Muro P., Monni S. e Tridico P. [2012], «L'evoluzione del modello socio-economico romano tra retorica e realtà», in POMPEO F. (a cura di), Paesaggi dell'esclusione. Politiche degli spazi, re-indigenizzazione e altre malattie del territorio romano, Milano, UTET.

DE Sio L. [2018], Il ritorno del voto di classe, ma al contrario (ovvero: se il PD è il partito delle élite), CISE LUISS, pubblicato on-line.

Di PAdova P. [2016], «Segnali dai margini. Un'analisi ecologica dei dati elettorali», in MORCELLINI et al. (2016).

DiLETTI M. [2016], «Roma, una città senza vocazione», in il Mulino, 2. 
Diletti M. e GRITTI R. [2016], «Metropolitics: elettori, sindaci e partiti a Roma (1993-2016)», in Morcellini et al. (2016).

LELO K. [2015], «Disuguaglianze socio-economiche e trasformazioni urbane nella Roma contemporanea (fine XX - inizi XXI secolo)», in Roma Moderna e Contemporanea, 23, 1-2, pp. 291-328.

Lelo K., Monni S. e Tomassi F. [2017], «Roma, tra centro e periferie: come incidono le dinamiche urbanistiche sulle disuguaglianze socio-economiche», in Roma Moderna e Contemporanea, 25, 1-2: 131-146.

Lelo K., Monni S. e TOMASsi F. [2018], «Disuguaglianze metropolitane: un confronto con Milano e Napoli», in D'AlBERGo E. e DE LEO D. (a cura di), Politiche urbane per Roma. Le sfide di una Capitale debole, Roma, Sapienza University Press.

MaCCAGNO A. [2018], Addio zone rosse: il centrosinistra si rinchiude nei centri urbani, YouTrend, pubblicato on-line.

MARCHIANÒ F. [2015], La competizione elettorale intrapartitica nelle comunali a Roma. Voto di preferenza e micropersonalizzazione, XXIX Convegno SISP, Cosenza.

Morcellini M., FagGiano M.P. e Nobile S. (a cura di) [2016], Dinamica Capitale. Traiettorie di ricerca sulle amministrative 2016, Santarcangelo di Romagna, Maggioli.

NATAlizia G. e Vignati R. [2016], «Roma: una svolta annunciata», in VAlBruZzi M. e VignATI R. (a cura di), Cambiamento o assestamento? Le elezioni amministrative del 2016, Bologna, Istituto Cattaneo.

Passarelli G. e VAlBruZzi M. [2018], Elezioni regionali nel Lazio 2018. Chi ha vinto, chi ha perso, Istituto Cattaneo, pubblicato on-line.

Rossi U. [2018], «The populist eruption and the urban question», in Urban Geography, 39, 9, pp. 1425-1430.

SEllers J.M., KÜBler D., WAlter-RogG M., Walks R.A. (eds.) [2013], The political ecology of the metropolis, Colchester, ECPR Press.

SELMINI R. [2016], «Ethnic conflicts and riots in Italy: The case of Rome, 2014», in European Journal of Criminology, 13, 5, pp. 626-638.

TOCCI W. [2008], «La città del tram», in TocCI W., INSOLERA I. e Morandi D., Avanti c'è posto. Storie e progetti del trasporto pubblico a Roma, Roma, Donzelli.

ToccI W. [2015], Roma, non si piange su una città coloniale. Note sulla politica romana, Firenze, GoWare.

TOMASSI F. [2013], «Disuguaglianze, beni relazionali ed elezioni nelle periferie di Roma», in Rivista di Politica Economica, 1-3, pp. 403-450.

TOMASSI F. [2015], «Dinamiche elettorali a Roma 2000-2014», in Tocci (2015). 
Tomassi F. [2018], Changes in the Eternal City: inequalities, commons and elections in Rome districts from 2000 to 2018, working paper.

TRUGLiA F.G. [2015], «Roma elettorale. Lo spazio geografico del consenso politico», in Cipollini e Truglia (2015).

VIOLANTE A. [2008], La metropoli spezzata. Sviluppo urbano di una città mediterranea, Milano, Franco Angeli.

ViOLANTE A. [2013], «Piove sul bagnato. La povertà urbana in una periferia metropolitana ai tempi della crisi», in Rassegna Italiana di Sociologia, 54, 2, pp. 279-308. 